\title{
THE PANDEMIC SCENARIO AND BONE MARROW TRANSPLANTATION
}

\author{
Erika Oliveira de Miranda Coelho \\ Hospital Santa Joana, Recife/PE
}

Correspondence to: erikaodmcoelho@hotmail.com

The COVID19 pandemic scenario has had a significant impact on the world economy and health. Restrictive measures of social distancing to contain the advance of contamination caused health services the need to review their care protocols and their transplant procedures. The pandemic has brought to light the precariousness of health services and the increased barriers to access health care causing some other problems.

Maintaining bone marrow transplantation activities was a major challenge, as hematological diseases require prompt treatment. Efforts were needed to redefine indications, workflows, hospitalization and isolation. It was essential to have the ability to test our patients, donors and professionals for COVID19. It was also important to ensure that health professionals did not transmit the virus to their patients even without fully understanding the virus's kinetic behavior. Above all, it was extremely important to have a support of an infectologist, and their increasing expertise, acquired almost daily, that could help patients with prevention strategies and care, besides understanding the aggressiveness and diversity of clinical expressions of this disease.

Our patients had to face the fear and uncertainties of their hematological disorders and now also the risks of COVID19. Many of their doubts still has no answer. Social distancing has put another point of stress in this scenario causing more suffering. The effects of the pandemic on the mental health to all of us was an important point to address and treat.
Over the months, which seemed years, we realized that the knowledge gained was unprecedented and made possible to minimize the devastating impact of the virus on our patients. Telemedicine has helped us, as never before, in interacting with other fellow hematologists and their medical and personal experiences around the world. We have never felt so vulnerable as professionals in the face of such a devastating disease and never needed so much interaction and trust in each other before. We learned a lot about hematology, bone marrow transplantation, immunology and inflammatory response but in times of pandemic and health crisis we learned even more about empathy, hope and gratitude.

\section{REFERENCES}

1. Recommendations of Sociedade Brasileira de Transplante de Medula Óssea (SBTMO)

2. Recommendations of American Society for Transplantation and Cellular Therapy (ASTCT)

3. Recommendations of European Bone Marrow Transplantion (EBMT)

4. Guan WJ, Ni ZY, Hu Y, Liang WH, Ou CQ, He JX, et al. Clinical characteristics of coronavirus disease 2019 in China. New Engl J Med. 2020,v.382,n.18, p.:1708-20.

5. Liang W, Guan W, Chen R, Wang W, Li J, Xu K, et al. Cancer patients in SARS-CoV-2 infection: a nationwide analysis in China. Lancet Oncol. 2020, v.21, n.3, p.:335-7. 